\title{
CONSIDERAÇÕES SŌBRE O TRATAMENTO DAS HEMORRAGIAS SUBARACNÓIDEAS ESPONTÂNEAS
}

\author{
J. Armbrust-Figueiredo *
}

As hemorragias subaracnóideas espontâneas são síndromes freqüentemente encontradas em clínica neurológica. Até há alguns anos seu tratamento era incompleto e insuficiente. Porém, com o aperfeiçoamento de métodos diagnósticos e com a adoção de novas técnicas cirúrgicas, seu tratamento se tem tornado muito mais eficaz e seu prognóstico menos grave.

Por hemorragia subaracnóidea espontânea se entende a extravasão não traumática de sangue no espaço subaracnóideo; constitui um sintoma no curso de processos intracranianos. E', na grande maioria dos casos, devida à rotura de um aneurisma arterial intracraniano. Raros são os casos em que é resultado de outros processos, como acidentes cerebrais hipertensivos, doenças vasculares ou tumores hemangiomatosos intracranianos.

A correta orientação no problema dos aneurismas intracranianos é devida, principalmente, a Dandy ${ }^{1}$, com a publicação de sua monumental monografia sôbre o tratamento cirúrgico dessas anomalias vasculares.

\section{INCIDENCIA}

Os aneurismas são encontrados em 0,5 a $1,5 \%$ das autópsias gerais ${ }^{2,3}$ e, devido às dificuldades em seu diagnóstico, freqüentemente não são identificados clìnicamente. Moritz e Zamchek ${ }^{4}$, em uma série de mil casos de mortes repentinas em soldados jovens, encontraram que, em 6,9\%, eram devidas a hemorragias provocadas pela rotura de um aneurisma intracraniano.

As localizações mais freqüentes dos aneurismas são as bifurcações dos grandes vasos da base do cérebro, tais como a junção da carótida interna com a cerebral média ou com a cerebral anterior. McDonald e Kork ${ }^{5}$, em 1.023 casos de aneurismas intracranianos (fig. 1), encontraram, em $72 \%$

'T'rabalho apresentado à Conferência de Neurologia e Neurocirurgia da George Washington e Georgetown Medical Schools, Gallinger Hospital, $\mathrm{fm} 24$ de janeiro de 1952 .

* Assistente de Neurologia na Escola Paulista de Medicina (Serviço do Prof. Paulino Longo). Assistente de Neuropatologia da George Washington University School of Medicine (Serviço do Prof. Walter Freeman) e Assistente-Residente de Neurologia no Gallinger Municipal Hospital, Washington, D.C. 
dos casos, localização na artéria carótida interna ou em seus ramos. O fato de que apenas $28 \%$ dos aneurismas são encontrados posteriormente significa que, na maioria dos casos, o tratamento cirúrgico é possível.

Em relação à idade dos pacientes, a incidência é maior entre os 30 e 50 anos. Contudo, casos de aneurismas em crianças têm sido descritos ${ }^{6}$.

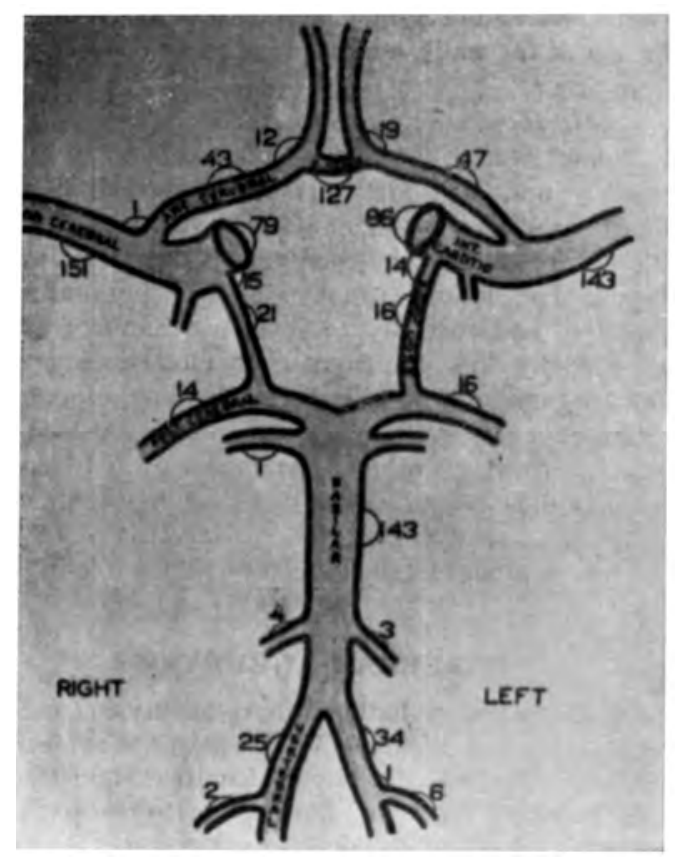

Fig. 1 - Localização de aneurismas intracranianos em 1.023 casos (McDonald e Korb 5).

\section{ETIOLOGIA}

Os aneurismas intracranianos parecem ser devidos a um imperfeito desenvolvimento das paredes vasculares, nos primeiros estádios embrionários. Estudando cortes microscópicos seriados de 13 casos de aneurismas intracranianos não inflamatórios, Carmichael ${ }^{7}$ encontrou hipoplasia vascular associada a uma degeneração arterial. Dêstes 13 casos, 7 não se haviam rompido; 11 casos envolviam a artéria carótida interna ou seus grandes ramos e em 2 casos os aneurismas situavam-se na junção da artéria cerebelar póstero-inferior com a artéria vertebral. $\mathrm{O}$ estudo histológico demonstrou lesões nas camadas muscular e clástica, sendo que, na camada média, se encontrava aplașia ou hipoplasia do tecido, às vêzes acompanhada de lesões degenerativas. A lesão da membrana elástica era devi- 
da a alterações degenerativas apenas, em geral relacionadas a um processo ateromatoso comum. Não foi possível a diferenciação entre os chamados aneurismas congênitos e os aneurismas arterioscleróticos. A incidência dos aneurismas intracranianos estaria, pois, diretamente relacionada à coexistência de lesões nas camadas íntima e média, o que ocorreria ao acaso.

A sífilis como agente causal de aneurismas é destituída de importân. cia. Émbolos micóticos podem produzir aneurisınas, quando a infecção penetra nas paredes vasculares, destruindo as camadas elásticas e muscular, que são substituídas por tecido fibroso pouco resistente. Geralmente, contudo, constituem falsos aneurismas, formados pela organização da camada mais externa de um hematoma.

\section{SINTOMATOLOGIA}

Os aneurismas geralmente não provocam sintomas clínicos antes de sua rotura. Entretanto, na dependência de seu tamanho e localização, e devido à compressão das estruturas vizinhas, podem causar o aparecimento de sinais neurológicos. Essa compressão atinge comumente alguns dos pares cranianos, resultando paralisias dos movimentos oculares, defeitos dos campos visuais, dor na face, etc. Quando localizados na região periquiasmática, por exemplo, podem ocasionar congestão venosa e conseqüente hiperemia e edema da papila óptica.

A cefaléia está muitas vêzes presente longo tempo antes da rotura do aneurisma, ocasionalmente imitando enxaqueca (migraine). A cefaléia é devida, em geral, a um aumento da pressão intracraniana ou pressão local do aneurisma contra as meninges ou algumas das raízes sensitivas.

Na grande maioria dos casos, porém, o aneurisma só é reconhecido pela hemorragia. E' um paciente que, em perfeito estado de saúde, sofre de uma crise aguda de cefaléia seguida por perda da consciência. A cefaléia, quando ocular, é excruciante, semelhante a uma facada, e devida à compressão do ramo oftálmico do nervo trigêmeo. Outras vêzes a dor se inicia na região occipital e na nuca, devido à irritação das meninges, e o paciente a descreve como se fôra um golpe de martelo. $O$ enfêrmo cai inconsciente, muitas vêzes apresentando convulsões epileptiformes; apresenta-se delirante; a respiração é do tipo Cheyne-Stokes, em geral devida à compressão dos centros bulbares; vômitos não são raros. Há rigidez da nuca e sinais de irritação meníngea aparecem (sinais de Kernig e Brudzinski). Os reflexos tendinosos mostram-se abolidos, geralmente. Quando recupera a consciência, na maioria dos casos, o enfêrmo apresenta paralisias de alguns dos nervos cranianos. A mais comum é a paralisia do nervo oculomotor; a paralisia facial é encontrada freqüentemente.

Os aneurismas da artéria vestebral podem simular um tumor do ângulo pontocerebelar ${ }^{8,9}$ com compressão do aqueduto de Sylvius e conseqüente dilatação de todo o sistema ventricular. A compressão do nervo 
óptico por aneurismas da carótida interna pode provocar o aparecimento de sintomas visuais por longo tempo, chegando mesmo a causar a síndrome de Foster-Kennedy ${ }^{10}$.

O exame do líqüido cefalorraquídeo revela aumento da pressão e líquor sangüinolento ou xantocrômico. Radiografias do crânio são negativas em quase todos os casos, exceção feita naqueles em que as paredes do aneurisma estão calcificadas (fig. 2). A encefalografia é de pouco auxílio, desde que o sistema ventricular apresenta apenas alterações mínimas, devidas principalmente à compressão da cisterna basilar, da cisterna interpeduncular ou da porção anterior do terceiro ventrículo pelo aneurisma ${ }^{11}$. A eletrencefalografia é de pouco ou nenhum valor diagnóstico; apenas nos casos com hemorragia intracerebral podem surgir lesões focais (caso 3).

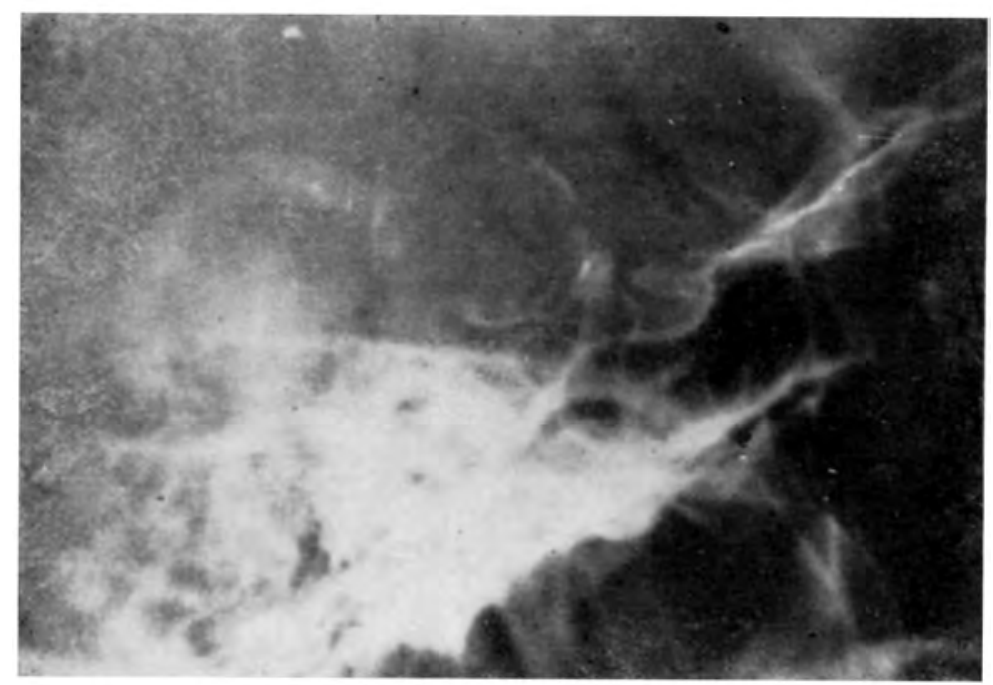

Fig. 2 - Radiografia do crânio mostrando grande ancurisma calcificado.

Em grande número dos casos a rotura do aneurisma não se faz apenas no espaço subaracnóideo, mas também no tecido intracerebral (casos 3 e 5). Estudando 93 casos de aneurismas, Robertson ${ }^{12}$ encontrou lesões intracerebrais em $65(69,9 \%)$, sendo que os aneurismas das artérias cerebral anterior e comunicante anterior tendem a sangrar na substância de um ou ambos lobos frontais̄ os da artéria cerebral média nos lobos temporal ou frontal; os da artéria cerebral posterior nos lobos occipital ou temporal ou nos gânglios da base; os da artéria basilar, em geral, causam apenas hemorragia subaracnóidea. 
Quando existe lesão intracerebral, os sinais de comprometimento dos centros superiores são mais evidentes. Hemiplegias, distúrbios da sensibilidade, afasia, etc., podem estar presentes.

\section{DIAGNÓSTICO}

O diagnóstico da hemorragia subaracnóidea $\dot{f}$ feito pelo exame do líqüido cefalorraquídeo (xantocrômico ou sangüinolento), e principalmente, pela arteriografia, que deve ser realizada logo que as condições do paciente permitam. A arteriografia tem sido descrita detalhadamente na literatura ${ }^{13,14,15}$.

As arteriografias são efetuadas sob anestesia, local ou geral. A anestesia geral com tionembutal (pentotal sódico) é a preferida, trazendo completo relaxamento do enfêrmo e evitando que se movimente em reação à introdução da substância radiopaca, que causa espasmo dos vasos cerebrais e dor. Esta reação é mais evidente quando se emprega o diodrast. As arteriografias sob anestesia local devem ser efetuadas apenas quando o gânglio simpático cervical superior e o seio carotídeo foram prèviamente bloqueados com procaína.

Os contrastes usados mais comumente são o diodrast e o torotrast (dióxido de tório coloidal); êste permite melhor imagem radiográfica, mas não deve ser usado quando a introdução da agulha se íaz através da pele, pois provoca necrose do tecido subcutâneo; assim, o torotrast é indicado apenas quando a artéria é exposta cirùrgicamente. Prèviamente ao uso de diodrast, provas de sensibilidade ao iodo devem ser efetuadas. Normalmente, são tomadas chapas de frente e perfil. Para melhor segurança, contudo, é indicado o śrteriograma bilateral, que facilita a avaliação da circulação colateral e a identificação de aneurismas múltiplos, o que se dá em $10 \%$ dos $\operatorname{casos}^{16}$.

Com a injeção do contraste nas artérias carótidas, a maioria dos casos é diagnosticada, pois cêrca de $78 \%$ dos aneurismas estão situados na própria carótida ou em seus ramos principais. Quando arteriografias bilaterais não demonstram a lesão, existe a possibilidade de o aneurisma estar localizado na artéria vertebral ou em um de seus ramos. Nesses casos, a injeção de contraste nas artérias vertebrais ${ }^{17}$ será necessária para fins diagnósticos. A introdução da agulha pode ser feita por via percutânea ou após dissecção do vaso. Este processo, contudo, é mais de valor acadêmico, desde que a remoção cirúrgica de aneurismas nas artérias vertebrais ou em seus ramos é em geral problemática; sua localização torna muitas vêzes o acesso cirúrgico impossível. Recentemente, contudo, um aneurisma localizado eñ um ramo anômalo da artéria basilar foi isolado cirùrgicamente ${ }^{18}$.

As arteriografias são, em geral, de pouco valor na presença de aneurismas miliares, mesmo quando múltiplos (caso 3 ). 


\section{PROGNOSTICO}

O prognóstico dos áneurismas intracranianos é grave. Segundo as estatísticas, o número de pacientes que morrem após a primeira hemorragia é, aproximadamente, de $40 \%$. Cêrca de $30 \%$ daqueles que resistem à primeira rotura, virão a falecer no segundo, terceiro ou quarto episódios, dentro de um período de 10 anos ${ }^{19}$. Daí a importância do tratamento cirúrgico na prevenção de novos acidentes hemorrágicos. $O$ fato de um paciente obter recuperação completa com repouso absoluto na cama não significa cura. Rosen e Kaufmann ${ }^{20}$ citam um caso de morte 27 anos após a primeira hemorragia e Strully ${ }^{21}$ um outro depois de 25 anos. 0 prognóstico é sempre mais grave quando o paciente é idoso, se há coexistência de hipertensão arterial e quando existe hemorragia intracerebral.

\section{TRATAMENTO}

Embora muitos sejam favoráveis à intervenção cirúrgica imediata nos casos de rotura de um aneurisma, o conceito atual é de que se deve agir cautelosamente, procurando dar ao paciente repouso absoluto e os cuidados de enfermagem necessários. Sedativos devem ser usados a fim de evitar tôda e qualquer agitação do paciente e prevenir nova hemorragia. Morfina e paraldeído são indicados como sedativos. A cefaléia deve ser controlada com compressas de gêlo, codeína ou qualquer outro dos medicamentos comumente usados. $O$ enfêrmo deve permanecer em repouso absoluto na cama pelo menos durante um mês depois da mais recente hemorragia. Confôrto moral é indispensável a êstes enfermos, devendo-se evitar qualquer choque emocional.

Diagnosticado o aneurisma, surge o problema da escolha do tratamento a ser empregado. Dois tipos distintos podem ser usados: o conservador ou o cirúrgico. O tratamento conservador é justificado apenas em algumas circunstâncias, como aneurismas intracranianos múltiplos bilaterais, aneurismas arterioscleróticos saculares com escassos sintomas e em casos em que inadequada circulação colateral não permita o tratamento cirúrgico.

O tratamento cirúrgico pode ser direto ou indireto. 0 tratamento indireto consiste na ligadura de uma ou mais das artérias do pescoço, usualmente a carótida interna, e tem a finalidade de diminuir a pressão do fluxo arterial dentro do aneurisma. Muitos autores consideram êste tratamento suficiente e o mais indicado para os diferentes tipos e localizações de aneurismas originados nos ramos $\mathrm{d}$ a carótida interna, pois assim é respeitada a integridade das paredes aneurismáticas já enfraquecidas ${ }^{22}$.

$\mathrm{O}$ tratamento cirúrgico direto foi introduzido por $\operatorname{Dandy}^{1} \mathrm{e}$ pode consistir em diferentes técnicas, como isolamento do aneurisma entre clips de metal aplicados nas partes distal e proximal da artéria, remoção do aneurisma, aplicação de músculo ao redor da dilatação aneurismática, incisão do aneurisma e colocação de músculo dentro da cavidade, etc. 
A escolha do tipo de tratamento a ser empregado varia em cada caso, dependendo de um estudo particular do aneurisma, sua localização, avaliação da circulação colateral, possibilidade de acesso cirúrgico ao vaso arterial e probabilidado de desenvolvimento de déficits funcionais secundários ao tratamento, tais como hemiplegias, afasia, etc. Nenhuma técnica pode ser indicada como regra geral e como a melhor, desde que os fatôres a serem considerados variam em cada caso. Cada enfêrmo é um problema distinto e particular.

De maneira geral, contudo, pode-se dizer que o tratamento cirúrgico indireto é o procedimento de escolha na maioria dos pacientes nos quais o aneurisma está localizado nas hifurcações das grandes artérias do círculo de Willis. Esse tipo de tratamento é contra-indicado quando a situação e conformação do aneurisma permitam o seu isolamento (casos 2 e 4) ou quando seja de pouco ou nenhum valor, o que acontece quando os aneurismas estão distantes do círculo de Willis.

O tratamento indireto pode ser levado a efeito pela oclusão parcial ou total das artérias carótidas comum, externa ou interna. A oclusão parcial é indicada naqueles casos em que a total não é bem tolerada pelo paciente. Nesses casos usa-se envolver a artéria em substâncias irritantes que venham a provocar o estreitamento do lume arterial, como celofane. fascia, ou ligaduras de sêda não muito apertadas. A ligadura total pode ser feita em uma ou mais das artérias carótidas. A oclusão da artéria carótida comum traz uma queda de aproximadamente $50 \%$ na pressão do fluxo sangüíneo e representa boa técnica para $o$ tratamento indireto. Se a circulação do lado oposto é considerada suficiente, a ligadura da caró. tida comum pode ser seguida pela oclusão da varótida externa acima da origem da artéria maxilar externa, permitindo assim a integridade funcional de vários ramos que irão fornecer sangue à carótida interna. A ligadura da carótida interna é aquela que fornece melhores resultados em ge. ral. E', também, um processo mais seguro, desde que na carótida comum são encontradas mais freqüentemente placas ateromatosas que podem provocar a rotura da íntima com conseqüente formação de trombo local. Quando ligada a carótida interna, a circulação é mantida por meio das artérias comunicantes anterior e posterior.

O material usado para a oclusão dos vasos ao nível do pescoço varia de acôrdo com cada autor. Sutura de sêdu, clips de metal, tubo de polietileno, etc., são usados. Temos empregado clip̣s especiais de tântalo, que apresentam a vantagem de poderem ser removidos fàcilmente se o enfêrmo apresentar sinais de intolerância à oclusão da artéria. (caso 2).

Certas precauções devem ser tomadas durante e após a ligadura dos vasos do pescoço, a fim de evitar complicações posteriores. A pressão arterial deve ser mantidia constante e dentro de limites normais; oxigênio deve ser administrado em quantidade adequada nas primeiras 24 a 48 horas; terapia anticoagulante deve ser instituída por 3 a 4 dias, a fim de 
evitar formação de êmbolos; bloqueio do gânglio cervical superior deve ser realizado com procúna, com a finalidade de impedir o espasmo arterial. A fim de evitar o aparecimento da síndrome de Horner, temporária ou permanente, recomenda-se o emprêgo de instilação contínua de procaína através de um tubo de polietileno suturado à bainha do gânglio cervical superior ${ }^{23}$.

Acidentes conseqüentes ao tratamento indireto não são raros. $\mathrm{Na}$ estatística de Poppen ${ }^{23}$ - uma das maiores e mais completas na literatura e na qual estão incluídos 143 casos de aneurismss tratados cirùrgicamente - o tratamento indireto foi usado em 101 enferrnos, com 3 casos de morte imediata, 5 casos de morte tardia devido a rotura do aneurisma e 8 casos de hemiplegia.

O tratamento direto, que consiste no ataque cirúrgico ao aneurisma, é geralmente reservado para os casos em que o aneurisma não se origina em uma das bifurcações dos grandes vasos da base do cérebro, a não ser que exista um pedículo de tamanho suficiente para a aplicação de um clip metálico, sem interferir com a circulação principal. O tratamento direto deve ser sempre precedido pelo indireto, a fim de que o fluxo sangǘneo seja reduzido e que o trauma cirúrgico não provoque a rotura do aneurisma. Em certas ocasióes o tratamento intracraniano é combinado ao processo indireto, o aneurisma sendo envolvido em múscillo, celofane ou algodão, desde que esteja localizado próximo à bifurcação da carótida interna ou quando a rotura se deu dentro do seio cavernoso.

A colocação de clip metálico no pedículo de um aneurisma é muitas vêzes usada, mas não representa processo inteiramente seguro, visto que a ohstrução é feita em local onde a parede do vaso é fraca, onde a camada média é incompleta ou ausente e, portanto, sede propícia de nova rotura. A excisão do aneurisma deve ser reservada para condições especiais e apenas quando o aneurisma está localizado distante do círculo de Willis. A técnica mais segura para o isolamento do aneurisma é a colocação de clips metálicos nas porções proximal e distal da artéria, eliminando o perigo de nova hemorragia no ponto onde a parede do vaso é deficiente.

A técnica operatória para exposição do círculo arterial de Willis é exatamente idêntica àquela usada para os tumores da hipófise. Feito um retalho ósseo, transfrontal ou frontotemporal, é êle refletido, prosseguindo-se na dissecção extradural em direção da asa do esfenóide. A região infrafrontal do cérebro é exposta por meio de incisão da dura ao longo da asa do esfenóide e em seguida em direção ao sulco olfatório. Esta ma. nobra permite a identificação dos vasos $\mathrm{d} a$ base do cérebro. A retirada de líqüido céfalorraquídeo, por meio de um dreno de polietileno na região lombar, com conseqüente diminuição da pressão intracraniana, permite melhor exposição dos casos.

Temos sempre procurado empregar ambos os tratamentos, direto e inreto, conjuntamente. Depois de diagnosticado o aneurisma e de estudada 
a suả localização, fazemos a oclusão da artéria carótida comum. Se houver boa tolerância, sem aparecimento de sinais neurológicos, ligamos a carótida interna, 7 a 10 dias após a primeira operação. E desde que indicado, em um terceiro estádio, 1 a 2 semanas mais tarde, levamos a cabo o tratamento intracraniano, com isolamento do aneurisma. Esse isolamento é feito, sempre que possível, entre dois clips de metal aplicados à artéria onde está localizado o aneurisma.

\section{RESLLLTADOS}

Reunindo as estatísticas de 37 autores e perfazendo um total de 469 casos operados, Mount ${ }^{16}$ encontrou uma mortalidade de $14 \%$, enquanto que, em 752 casos tratados pelo método conservador, a fatalidade foi de $48 \%$. Em sua própria estatística de 77 casos operados, Mount obteve, em $71 \%$, melhora com o tratamento cirúrgico; $14 \%$ não apresentaram melhoras, $4 \%$ pioraram e $11 \%$ faleceram. Dêstes 77 pacientes, 21 sofreram intervenção intracraniana, sendo que $86 \%$ melhoraram e $14 \%$ faleceram.

Se a operação pode prevenir nova hemorragia e morte do paciente na maioria dos casos, não resulta obrigatòriamente no desaparecimento dos sintomas. A cefaléia greralmente regride, mas as paralisias oculares, distúrbios visuais, hemiparesias e outros sintomas jodem persistir apesar do. tratamento cirúrgico (casos 2 e 4 ).

\section{OBSERVAÇOES}

Com o intuito único de ilustrar as dificuldades do tratamento dos aneurismas intracranianos, apresentamos cinco casos que tivemos a oportunidade de acompanhar recentemente. A sintomatologia das hemorragias subaracnóideas espontâneas, seu diagnóstico, a gravidade do prognóstico e a necessidade de rápido e adequado tratamciio cirúrgico dos aneurismas são bem evidenciados nesses casos.

Caso 1 - M. J. G., branca, com 33 anos de idade. Adnitida ao George Washington Hospital em 17-1-1951 (Reg. 67500). A paciente foi recebida no Serviço de Emergência em coma. Por vários dias a paciente se queixava de moderada cefaléia, que, contudo, não impediu suas atividades norniais. No dia da admissão, a paciente sùbitanente perdeu a consciência e entrou $\mathrm{cm}$ coma, enquanto fazia compras em uma loja e de onde foi transferida imediatamente para o hospital. A história pregressa revelava um ataque semelhante 4 anos antes, o qual roi diagnosticado como hemorragia subaracnóidea. A paciente permanecera inconsciente por três semanas, apresentando hemiplegia esquerda. Gradualmente melhorara, permanecendo em repouso absoluto no leito por dois meses. A hemiplegia aos poucos desaparecera e desde então a paciente se queixava apenas de ocasionais crises de cefaléia, mais comuns durante o período nienstrual.

Exame - Haciente em coma, com pulso rápido e respiração difícil. A pressão arterial não pôde ser obtida. $O$ exame de urina revelou traços de albumina. $O$ exame químico do sangue mostrou $154 \mathrm{mg} \%$ de glicose e $13 \mathrm{mg} \%$ de nitrogênio urêico. A enfêrma foi colocada em um respirador, com o que foi possível 
slevar a pressão arterial a 100-75. Contudo, a paciente permaneceu comatosa, vindo a falecer na manhã do dia seguinte.

A autópsia revelou hemorragias subaracnóidea e subdural no hemisfério esquerdo e base do cérebro. Na face inferior do lobo frontal, no giro reto direito, foi encontrado um grande aneurisma medindo $1 \times 3 \mathrm{~cm}$. Após fixação em forrnol, o cérebro foi seccionado sagitalmente na linha média, mostrando o aneurisma na artéria cerebral anterior direita, envolvido por coágulo sangiíneo. $O$ tecido cerebral ao redor se apresentava hemorrágico, com áreas de necrose e enfarte. Os terceiro e quarto ventrículos se mostravam completamente cheios de sangue (fig. 3).

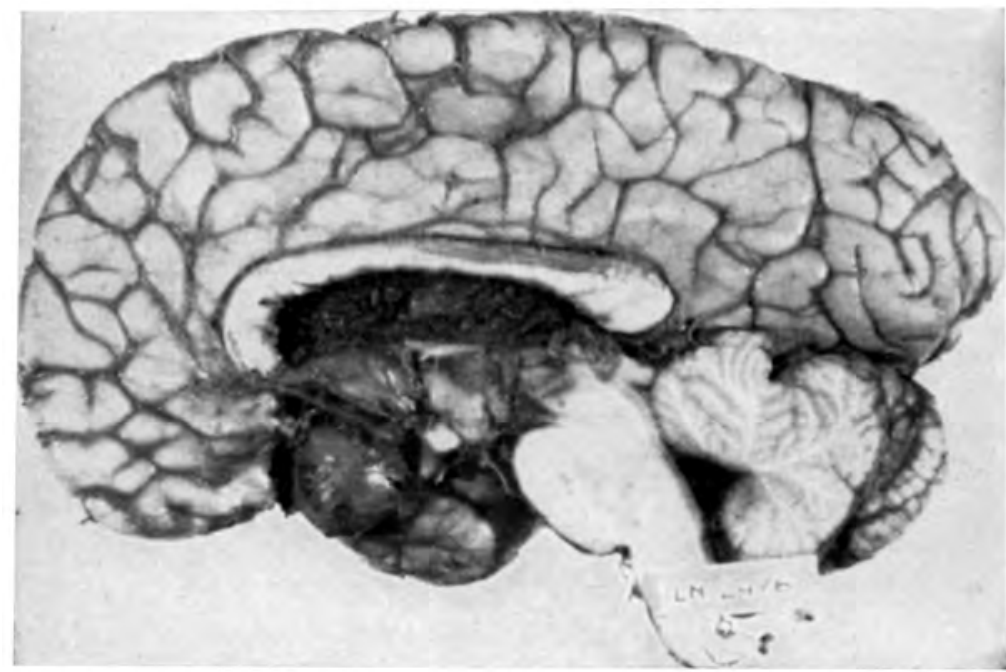

Fig. 3 - Caso 1 (M. J. G.). Grande aneurisma da artéria cerebral anterior, cuja rotura provocou destruição da face inferior do lobo frontal e inundação do sistema ventricular.

Caso 2 - Janie C., preta, com 37 anos de idade. Admitida ao Gallinger Municipal Hospital em 11-8-1951 (Reg. D-59427). A paciente veio ao ambulatório dentário nessa data, queixando-se de parestesias e cefaléia na região frontal. Essa sintomatologia surgiu repentinamente duas semanas antes da admissão; depois de alguns dias tornou-se mais severa, envolvendo também o lado esquerdo da face e região periauricular. A paciente procurou, então, um dentista, que aconselhou e removeu um dos dentes molares. Entretanto, a parestesia tornou-se mais intensa e no dia 8 de agôsto a paciente se tornou confusa e desorientada. Surgiram paralisia facial esquerda e estrabismo do ôlho esquerdo. Quando vista no serviço dentário, a paciente estava sonolenta e desorientada. Foi-nos solicitado o exame neurológico.

Exame -- Paciente falando confusamente e scmicomatosa. A pressão arterial era 110-60, pulso 60 bat./min, respiração $16 \mathrm{mov} . / \mathrm{min}$. Aparelhos respiratório, circulatório e abdome normais. $O$ exame neurológico evidenciou ptose da pálpebra superior esquerda; olho esquerdo desviado externamente; pupila esquerda dilatada, não reagindo à luz ou à acomodação; pupila direita normal; hipoestesia 
da face esquerda; demais nervos cranianos intactos; não estavam presentes refiexos patológicos; a sensibilidade não pôde ser avaliada devidamente.

A paciente foi admitida ao Serviço Neurológico com a impressão de hemorragia subaracnóidea devida a rotura de aneurisma intracraniano. Uma punção lombar foi efetuada nesse mesmo dia, mostrando pressão inicial de $420 \mathrm{~mm}$ de água; líquor sangüinolento; $88 \mathrm{mg} \%$ de glicose; $71,42 \mathrm{mg} \%$ de proteínas; teste específico para lues (Kolmer) negativo. Hemograma: 4.800 leucócitos e 3.400 .000 hemácias; hemoglobina $71 \%$. O exame de urina fol normal. Testes serológicos para sífilis (Kahn e Kolmer) fortemente positivos. A 21 de agôsto um arteriograma foi obtido, revelando grande aneurisma na artéria carótida interna esquerda. imediatamente untes da origgen da cerebral méilia (fig. 4).

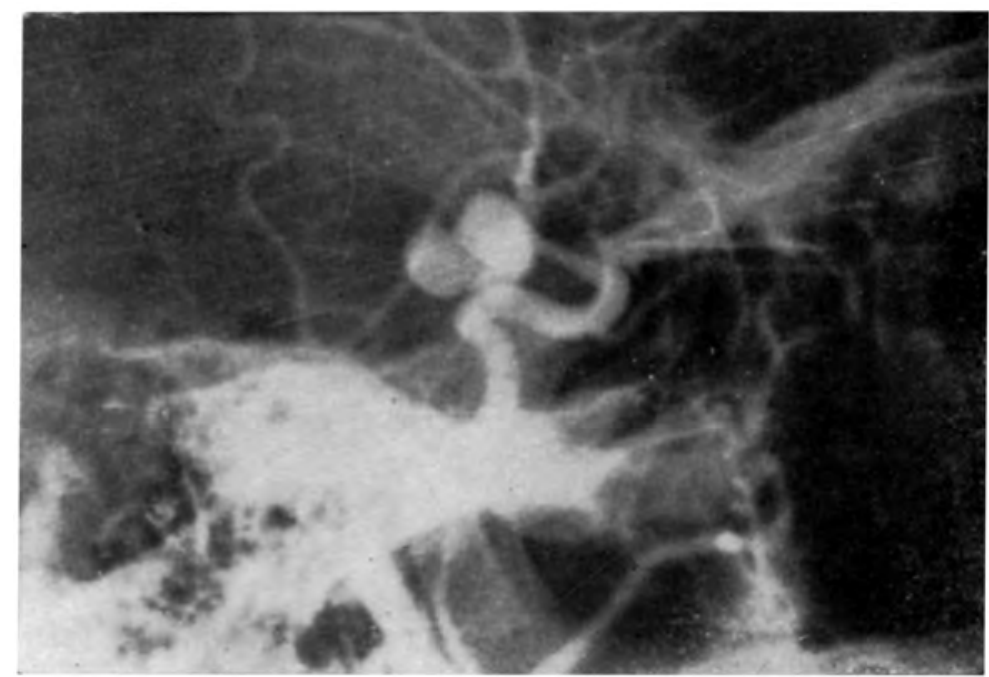

Fig. 4 - Caso 2 (J. C.). Grande aneurisma da artéria carótida interna evidenciado por arteriografia.

A 5 de setembro foi feita a ligadura da carótida comum e, uma semana mais tarde, a oclusão da carótida interna. A dissecção da artéria nessa segunda operação foi bastante difícil devido à presença de tecido fibroso cicatricial. Durante a manipulação cirúrgica a veia jugular interna foi atingida e, em virtude de profuso sangramento, foi decidida a sua ligadura. A carótida interna foi eritão clissecada e um clip de tântalo aplicado à sua parede. A paciente tolerou bem o processo. Contudo, 40 minutos depois, começou a apresentar sinais de afasia e hemiparesia direita. O clip foi removido e quase que imediatamente a paciente recuperou a fala normal e melhorou a paresia. Bloqueio do gânglio cervícal superior e terapia anticoagulante iniciada. A 19 de setembro a paciente foi levada novamente à sala cirúrgica e a incisão do pescoço foi reaberta. Durante a disseç̧ão a paciente apresentou crise convulsiva, que se iniciou na mão direita, abrangendo o lado direito e depois se tornando generalizada. Pela administração intravenosa de fenobarbital cessou a convulsão. A carótida interna foi então envolvida em celofane. A paciente tolerou bem o processo cirúrgico e, com a finalidade de evitar novas convulsões, foi instituído tratamente com dilantin e fenobarbital. Én virtude da localização do aneurisma e da presença de um pedículo 
bastante longo, foi decidido o isolamento intracraniano do aneurisma pela aplicação de um clip metálico no pedículo. A 7 de novembro, sob anestesia geral, uma craniotomia frontotemporal esquerda foi feita. Incisada a dura, conseguiu-se boa exposição dos lobos temporal e frontal, bem como da asa do esfenóide. Contudo, o cérebro se apresentava muito congesto e a coagulação e secção das veias ao longo da asa do esfenóide foram feitas com dificuldade. A apófise clinóide foi identificada, mas o nervo óptico e a carótida não puderam ser visualizados devido ao edema cerebral, que se acentuava com o trauma cirúrgico. Devide à possibilidade de um rotura do aneurisma em se forçando a exposição do círculo de Willis, foi decidida a interrupção da operação, para ser prosseguida posteriormente. A 12 de dezembro a craniotomia foi repetida, usando-se a mesma incisão. O cérebro apresentou-se normal e a identificação do nervo óptico $e$ da carótida interna foi feita fàcilmente, com afastamento dos lobos frontal e temporal. Um aneurisma foi encontrado na artéria carótida interna, imediatamente antes da origem da cerebral média, localizado quase totalmente sob o tentório. Um clip de prata foi aplicado à porção proximal da artéria; quando se tentava remover o clamp após a colocação de um outro clip à jorção distal da artéria, esta começou a sangrar profusamente, em virtude de o clip ter ficado prêso ao clamp. Êste foi finalmente removido e se pôde verificar que a carótida havia sido lacerada quase que totalmente. Dois clips foram aplicados à porção distal e a hemorragia controlada. A exploração mostrou as demais estruturas intactas e a incisão foi fechada. A paciente tolerou bem o processo cirúrgico, não desenvolvendo qualquer paralisia ou déficit motor. No dia 3 de janeiro foi dispensada e um exame neurológico nessa ocasião apenas evidenciou paralisia do terceiro par à esquerda. Vista por nós novamente a 1 de fevereiro, apresentava melhora da ptose e os movimentos do olho esquerdo eram normais.

Caso 3 - Susie F., preta, com 38 anos de idade. Admitida ao Gallinger Municipal Hospital em 8-10-1951 (Registro D-63517). A paciente foi vista por nós no Serviço de Emergência, com história de convulsões desde a noite anterior à admissão. A paciente estava perfeitamente bem até o momento em que se foi deitar, quando apresentou uma convulsão, iniciada por desvio dos olhos e da bôca para a direita, e movimentos tônico-clônicos do hemicorpo direito, generalizando-se depois a crise. Uma ambulância foi chamada e o médico interno administrou amital sódico intravenosamente, o que fêz cessar a convulsão. Na manhã seguinte a paciente acordou confusa e desorientada, falando incompreensivelmente e não se recordando dos acontecimentos da noite anterior. Pouco depois apresentou nova crise convulsiva, semelhante à anterior, e foi trazida para o hospital.

Exame - No Serviço de Emergência se mostrava semicomatosa, com pressão arterial 130-70, pulso 120 bat./min; respiração $16 \mathrm{mov} . / \mathrm{min}$. O exame dos pulmões, aparêlho cardiovascular e abdome foi negativo. $O$ exame neurológico mostrou os nervos cranianos intactos; os reflexos tendinosos se apresentavam hiperativos à direita e normais à esquerda; Babinski presente à direita; tono normal; reflexos abdominais ausentes; sensibilidade e cerebelo não puderam ser examinados. Enquanto examinada, a paciente apresentou nova crise convulsiva, semelhante às demais. A paciente foi admitida ao Serviço Neurológico com impressão de possível lesão intracraniana. 'Tratamento antiepilético foi instituído. No dia seguinte a paciente se apresentava com rigidez de nuca e ambas as regiões parotideas inchadas. Uma punção lominar foi feita nesse dia, mostrando pressão inicial de $400 \mathrm{~mm}$ de água, liquiido sangüinolento. Um eletrencefalograma tirado nesse dia evidenciou "spikes" bipolares na região temporal esquerda e ondas lentas de $5 \mathrm{c} / \mathrm{s}$ generalizadas, porém mais constantes nas regiões occipitais; os achados foram sugestivos de irritação cerebral generalizada e provável toco temporal. A paciente não apresentou outras crises convulsivas, mas se queixava de cefaléia e permanecia desorientada. 
A 12 de outubro a inchação das glândulas parótidas se mostrava mais acentuada e a hipótese de encefalite foi levantada. A rigidez da nuca permanecia. $O$ exame neurológico revelou fundus normal, pupilas iguais, reagindo à luz; reflexos hiperativos à direita; Babinski presente à direita; clono patelar à direita; sinal de Kernig. Nos dias subseqüentes a paciente se tornou mais letárgica e nova punção lombar mostrou líqüido xantocrômico com pressão inicial de $200 \mathrm{~mm}$ de água. A 27 de outubro a paciente se apresentava consciente e melhor orientada. Dois dias após, arteriograma percutâneo bilateral foi efetuado; não foi evidenciado qualquer aneurisma. $O$ estado geral da paciente melhorou acentuadamente e os reflexos tendinosos à direita voltaram a ser quase que norirais; o sinal de Babinski continuava presente à direita.

No dia 7 de novembro a paciente podia andar sem qualquer queixa. Ness? mesmo dia, à tarde, a paciente apresentou nova crise convulsiva generalizada con perda da consciência. A pressão arterial era de $110-80$, pulso $40 \mathrm{bat} . / \mathrm{min}$. As pupilas tornaram-se punctiformes, não reagindo à luz; os olhos desviaram-se para a direita; os reflexos tendinosos se apresentaram hiperativos em ambos os lados; sinal de Babinski bilateral. Uma punção lombar mostrou pressão inicial de 520 $\mathrm{mm}$ de água e líquor sangüinolento; removidos $10 \mathrm{ml}$ a pressão caiu a $320 \mathrm{rm}$. Logo após as pupilas se tornaram dilatadas e a paciente apresentou uma série de crises convulsivas generalizadas, que foram controladas pela administração de $600 \mathrm{mg}$ de fenobarbital. A paciente permaneceu inconsciente e surgiu ptose da pälpebra superior direita; os reflexos abdominais se tornaram ausentes. Oxigênio foi administrado por catéter nasal. A paciente expirou 3 horas após.

A autópsia revelou acentuada hemorragia subaracnóidea, com inundação des ventrículos. Extensa área de hemorragia intracerebral, com destruição do tálamo, núcleo caudado, globo pálido e cápsula interna, foi encontrada à esqueıda. A dissecção das artérias mostrou a presença de múltiplos aneurismas miliares.

Caso 4 - James H., preto, 38 anos de idade. Admitido ao Gallinger Municipal Hospital em 12-11-1951 (Reg. D-65906). 'Três semanas antes da admissão, o paciente estava deitado, ainda acordado, quando repentinamente teve forte crise de cefaléia, não localizada. A cefaléia permaneceu bastante intensa por uma semana, quando começou a regredir progressivamente. Nessa ocasião surgiu ptose da pálpebra superior direita e o paciente notou que não podia mover o ôlho direito enı direção à linha média. Procurou o Serviço de Émergêneia do Episiscopal Hospital, onde foi examinado pelo Dr. Jonathan Willians, o qual o encaminhou para o Gallinger Hospital.

Quando examinado por nós a 12 de novembro, se apresentava lúcido, bem orientado, com ptose da pálpebra superior direita e ôlho direito desviado externamente. Queixava-se de dor na região periorbitária e hemicrânio direitos. A pressão arterial era de 124-78, pulso 64 bat./nin. $O$ exame dos aparelhos respiratório e cardiovascular foi normal. $O$ exame neurológico mostrou a pupila direita maior que a esquerda, não reagindo à luz ou à acomodação; ptose da pálpebra superior direita; paralisia dos músculos inervados pelos $3^{\circ}$ e $4^{0}$ nervos à direita; fundus normal; demais nervos cranianos intactos; sistemas motor e sensitivo intactos; reflexos patelar e aquiliano hiperativos em ambos os lados; demais reflexos normais; cerebelo normal. $O$ paciente foi admitido ao serviço de Neurologia com diagnóstico de hemorragia subaracnóidea devida a rotura de aneurisma intracraniano.

Exame hematológico: 5.500 leucócitos, 4r\% hematócrito. Exame de urina, normal. 'Testes serológicos para sífilis negativos. Uma punção lombar foi îeita e mostrou pressão inicial de $270 \mathrm{~mm}$ de água, líquor sangüinolento, com 4.5,4.5 mg\% de proteinas e curva de ouro coloidal 011.100.000.00. A 14 de novembro um arteriograma foi obtído, revelando um aneurisma da artéria carótida interna, supraclinóideo (fig. 5). 


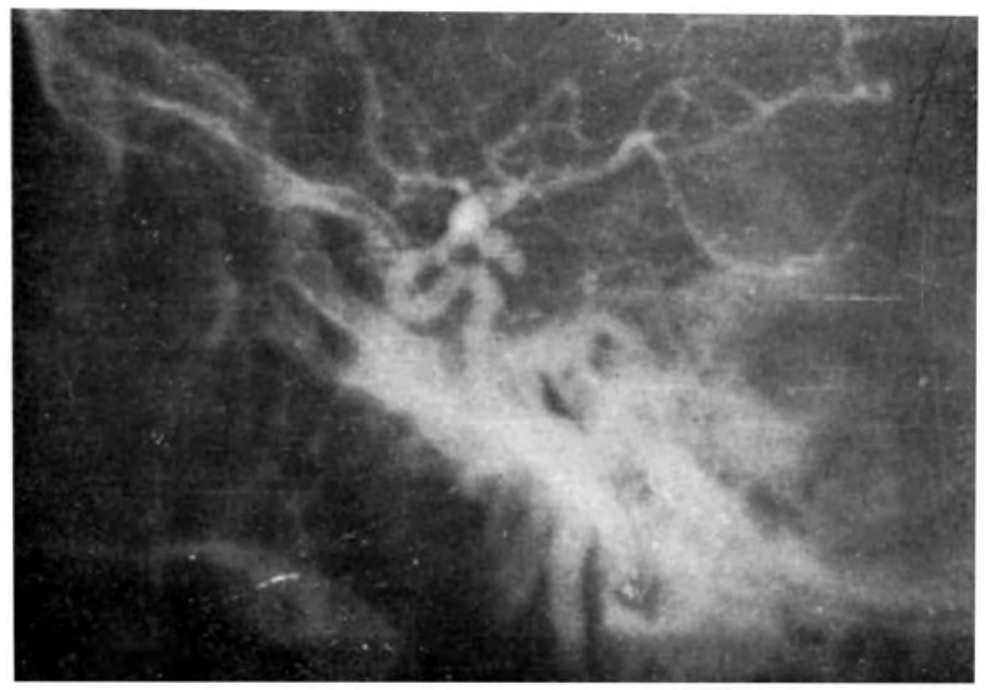

Fig. 5 - Caso 4 (J. H.). Arteriografia mostrando o anenrisma da artéria carótida interna.

Uszncio-se a mesma incisão feita para o arteriograma, fêz-se a ligadura da carótida comum. Avaliação do fluxo sangüíneo ccrebral foi obtida antes e depois da oclusão arterial. Antes da oclusão obtivemos um valor de $56,3 \mathrm{ml} / \mathrm{min} / 100$ $\mathrm{g}$ de cérebro; 15 minutos após a oclusão encontramos uma queda para 50,1 e depois de uma hora o valor era de 52,4. () paciente tolerou perfeitamente ben a ligadura da artérì. Uma semana mais tarde, $n$ nn clip de tântalo foi aplicedo a artéria carótida interna. A 28 de novembro, sob anestesia geral, uma craniotomia frontotemporal direita foi feita. Os lobos frontal e temporal foran afastados e todos os vas:s comunicantes situados na asa do esfenóide foram coagulados e seccionados. $O$ nervo óptice, o processo clinóide e a carótida interna foram identificados. O aneuríma foi encontrado na artéria carótida intema, sendo que parte do saco se achava sob o tentório. Dois clips metálicos foram aplicadcs à artéria, em suas partes distal e proximal, isolando completament. o aneurtsma e permitindo perfeita integridade da artéria cerebral média, que passou a receber sangue através das artérias comunicantes anterior e cerebral anterior.

No pós-operatório o paciente se apresenton letárgico, queixando-se de cefaléia frontal e demonstrando ligeira afasia motora (o paciente usa a mão esquerda). Gradativamente o estado do enfêrmo foi melhorando e a $\boldsymbol{t}$ de dezembro apenas fomos capazes de observar ptose da pálpebra direita, paralisia dos músculos oculares inervados pelos $3^{\circ}$ e $4^{\circ}$ nervos do lado direito e Babinski à esquerda. O paciente fó dispensado a 11 de dezembro e, quando visto por nós a 271-1952, era capaz de mover ligeiramente o ôlho direito em direção à linha média; a ptose da pálpebra superior direita era menos evidente, do modo a permitir que a fissura palpebral se apresentasse com metade do tamauho normal; a fala era perfeitamente normal.

Caso 5 - Bozella A., preto, com 45 anos de idade. Adwitido ao Galling $r$ Municipal Hospital em 27-12-1951 (Reg. D-68783). Êste paciente foi visto por 
nós pela primeira vez no Serviço de Moléstias Infecciosas, no dia de admissão. $O$ entêrmo apresentava história de moléstia hipertensiva por 18 anos, com ocasionais crises de cefaléia generalizada, que eram sempre controladas pelos medicamentos usuais. No dia 17 de dezembro o paciente queixou-se de forte cefaléia frontal e dor na nuca, o que o levou a abandonar o trabalho e permanecer em casa, a maioria do tempo recostado. A cefaléia persistiu por alguns dias, diminuindo em intensidade gradativamente. No dia 26 de dezembro o paciente queixou-se novamente de forte dor na nuca e minutos depois caiu ao solo inconsciente. Na manhã seguinte foi trazido ao Gallinger Hospital.

Exame - Quando visto por nós se apresentava letárgico, não respondendo a estimulos dolorosos, com rigidez da nuca; as pupilas se apresentavam em miose, não reagindo à luz; paralisia do $3^{\circ}$ nervo à direita; o exame funáoscópico foi negativo; os reflexos tendinosos se apresentavam hiperativos com clono da rótula $\mathrm{e}$ do pé em ambos os lados; Babinski à direita; os reflexos abdominais estavam ausentes. Admitimos o paciente ao Serviço de Neurologia com diagnóstico de aneurisma intracraniano.

Uma punção lombar foi feita e mostrou pressão inicial de $340 \mathrm{~mm}$ de água, líquor xantocrômico. Coração aunentado de volume, com sôpro diastólico no foco nitra!; pulmões e abdome liormais. Uma radiografia do crânio não mostrou anormalidades. $U$ exame de urina foi normal e o exame químico do sangue mostrou $569 \mathrm{mg} \%$ de cloretos, $49 \mathrm{mg} \%$ de proteínas e $18 \mathrm{mg} \%$ de nitrogênio urêico. $O$ paciente não recobrou a consciência e gradativamente as condições gerais foram-se tornando mais graves. Veio a falecer no dia 2 de janeiro.

A autópsia reveloil acentuada hemorragia subaracnóidea, mais intensa na região periquiasmática. O IV ventrículo se achava completamente obstruído por coágulo sanguíneo. O exame do círculo de Willis não evidenciou a presença de qualquer aneurisma. Após fixação em formol, o cérebro foi seccionado longitudinalmente na linha média, mostrando grande aneurisma àa artéria cerebral anterior direita, imediatamente anterior ao joelho do corpo caloso. Ventrículos completamente inundados (fig. 6).

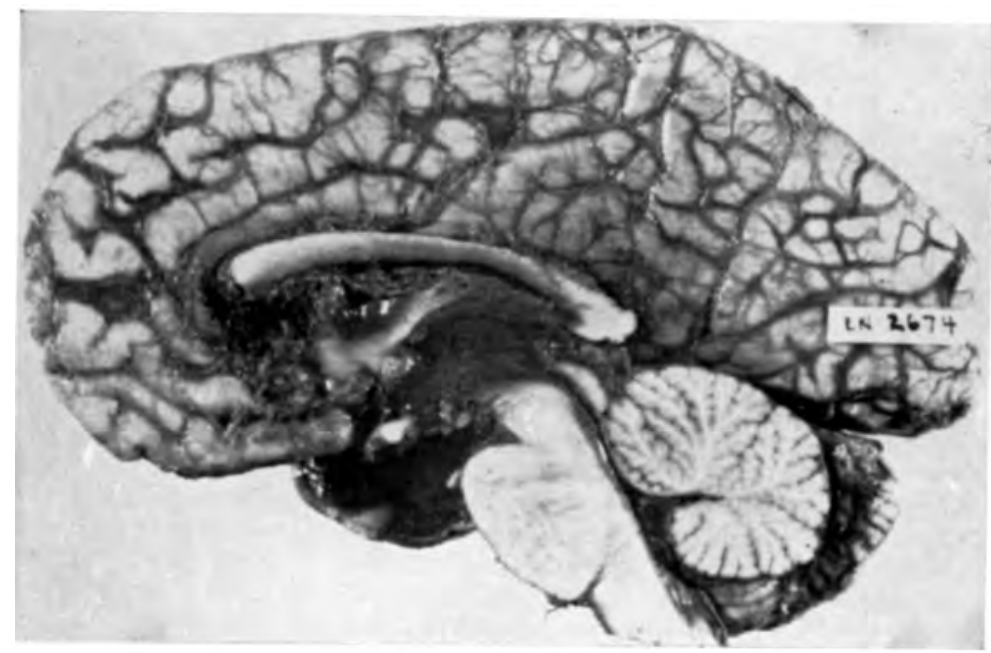

Fig. 6 - Caso 5 (B. A.). Aneurisma da artéria cerebral anterior localizado anteriormente ao joellio do corpo caloso; inundação dos ventrículos como resultado de sua rotura. 


\section{CONCLUSOES}

Estudando-se os diferentes métodos de tratamento dos aneurismas intracranianos e de acôrdo com as estatísticas encontradas na literatura, pode-se concluir que o tratamento conservador é indicado apenas em algumas circunstâncias especiais, como aneurismas intracranianos múltiplos bilaterais, aneurismas arterioscleróticos saculares com escassos sintomas e em casos em que a circulação colateral não é suficiente para manter o fluxo necessário ao hemisfério cerebral em aprêço. As estatísticas indicam que 48\% dos casos tratados paliativamente são fatais.

$\mathrm{O}$ tratamento cirúrgico, com mortalidade de $14 \%$ é, pois, indicado. A escolha da técnica ci:úrgica só poderá ser decidida depois de cuidadosa consideração sôbre as diversas circunstâncias que cada caso apresente, isto é, depois de serem estudadas a localização do aneurisma, a circulação colateral, a tolerância do paciente à oclusão de um ou mais vasos no pesccrço e a possibilidade de acesso cirúrgico. Sempre que possível, o tratamento cirúrgico deve associar o tratamento indireto ao direto, com oclusão de um ou mais vasos no pescoço seguida por craniotomia e isolamento do aneurisma. Embora com maior trauma cirúrgico, êsse procedimento traz maior segurança e diminui grandemente as possibilidades de nova hemor. ragia que, na maioria das vêzes, é fatal. O tratamento cirúrgico deve ser empregado sempre que as condições o permitam. Na maioria dos casos, se a cirurgia não fôr instituída, os pacientes virão a sofrer nova hemorragia.

\section{SUMARIO}

O tratamento das hemorragias subaracnóideas espontâneas é estudado pormenorizadamente. Com poucas exceçôes, êsses casos são devidos a rotura de um aneurisma intracraniano, situado em $72 \%$ dos casos na artéria carótida interna ou em um de seus ramos principais. A incidência e a etiologia dos aneurismas são discutidas. O aneurisma se deve a um imperfeito desenvolvimento das paredes vasculares, associado a uma lesão degenerativa. A sintomatologia está ausente, na grande maioria dos casos, até a rotura do aneurisma, quando o paciente perde a consciência, entra em choque, com sinais de comprometimento dos nervos cranianos vu dos centros superiores. A presença de sangue no líqüido cefalorraquídeo, com aumento da pressão, é achado diagnóstico. O prognóstico dos aneurismas é grave; $40 \%$ dos pacientes vêm a falecer com a primeira hemorragia; daqueles que resistem ao primeiro acidente, $30 \%$ virão a falecer no segundo, terceiro ou quarto episódios dentro de um período de 10 anos. 0 tratamento da fase aguda é conservador, com absoluto repouso no leito. Qıiando as condições o permitam, arteriografias devem ser obtidas para demons- 
tração do aneurisma. O tratamento cirúrgicc deve então ser instituído, a técnica variando de acôrdo com a localização da lesão, condições da circulação colateral, possibilidade de acesso cirúrgico direto, etc. 0 tratamento indireto deve ser associado ao direto, sempre que possivel: far-se-á, portanto, a oclusão de uma ou mais artérias no pescoço com subseqüente isolamento intracraniano do aneurisma. As diferentes técnicas cirúrgicas são discutidas. Nos casos tratados pelo método conservador a mortalidade é de $48 \%$ e nos casos tratados pela cirurgia, de 14\%. Cinco casos de aneurismas intracranianos são apresentados com a finalidade única de ilustrar os problemas clínicos. Esses casos demonstram os diferentes íipos de evolução clínica e refletem a gravidade do prognóstico dos aneurismas, mostrando a necessidade de imediato e adequado tratamento.

SUMMARY

The surgical treatment of spontaneous subarachnoid hemorrhage is presented in detail. In the great majority these cases are due to the rupture of an intracranial aneurysm, located in 72 per cent of the cases in one of the principal branches of the internal carotid artery. Incidence and etiology are studied. The aneurysms are the result of incomplete embryological development of the arterial wall associated with a degenerative lesion. The clinical signs are absent until rupture occurs. Patient loses consciousness, goes into shock and presents involvement of some of the cranial nerves or superior centers. The presence of bloody spinal fluid and increased intracranial pressure are findings suggesting the diagnosis. Prognosis is severe since 40 per cent of the patients die in the first attack. About 30 per cent of the remaining cases expire during the second, third or fourth episodes occurring during the next ten years. The management in the acute phase is essentially conservative with absolute bed rest. After some days, arteriograms must be obtained. If the aneurysm is shown, surgical treatment must be carried out. The surgical technique is chosen after careful study of the location of the lesion, the degree of colateral circulation and the possibility of intracranial surgical attack. The indirect treatment must be combined with the direct treatment when possible. Ligation of one or more arteries in the neck and subsequent trapping of the aneurysm should be done. The different types of surgical techniques are studied. The mortality rate for conservatively treated patients is 48 per cent and 14 per cent for those treated with surgery. Five cases of intracranial aneurysms are studied and presented as illustrations of the clinical problems. These patients showed different clinical course and reflect the seriousness of the syndrome and show the necessity for prompt and adequate treatment. 


\section{BIBLIOGRAFIA}

1. Dandy, W. L. - Intracranial Arterial Aneurysms. Comstock Publishing Co., Ithaca, Nova York, 1944.

2. Richardson, J. C. e Hyland, H. H. - Intracranial aneurysms; a clinical and pathological study of subarachnoid and intracerebral hemorrhage caused by berry aneurysms. Medicine, 20:1-83, 1941.

3. Courville, C. B. - Pathology of the Central Nervous System. A Study based upon a Survey of Lesions found in a Series of Forty Thousand Autopsies. Pacific Press, California, 1950.

4. Moritz, A. R. e 'Zamcheck, N. -- Sudden and unexpected deaths of young soldiers; diseases responsible for such deaths in World War II. Arch. Pathol., 42: $₫ 59-494$ (novembro) 1946.

5. McDonald, C. A. e Korb, M. - Intracranial aneurysm. Arch. Neurol. a. Psychiat., $42: 298-328,1939$.

6. Hermann, K. e MacGregor, A. R. - Cerebral haemorrhage from rupture of a congenital intracerebral aneurysm in a child. Brit. M. J., 1:523-525 (março) 1940.

7. Carmichael, R. - Pathogenesis of non inflammatory cerebral aneurysms. J. Path. a. Bact., 62:1-19 (janeiro) 1950.

8. Globus, J. H. e Schwab, J. M. - Intracranial aneurysms; their origin and clinical behaviour in a series of verified cases. J. Mt. Sinai Hosp., 8:547578, 1942.

9. Yaskin, H. E. e Alpers, B. J. - Aneurysm of the vertebral artery; report of a case in which the aneurysm simulated a tumor of the posterior fossa. Arch. Neurol. a. Psychiat., 51:271-281, 1944.

10. Ley, A. - Compression of the optic nerve by fusiform aneurysm of carotid artery. J. Neurol., Neurosurg. a. Psychiat., 13:75-86, 1950.

11. Davidoff, I. M. e Epstein, B. S. - The Abnormal Pneumoencephalogram. Lea and Febiger, Filadélfia, 1950.

12. Robertson, E. G. -- Cerebral lesions due to intracranial aneurysms. Brain, 72:150-185 (junho) 1949.

13. Moniz, E. - L’Angiographie Cérébrale. Masson et Cie., Paris, 1934.

14. Green, J. R. e Arana, R. - Cerebral angiography; a clinical evaluation based on 107 cases. Am. J. Roentgenol., 59:617-650, 1948

15. List, C. F., Burge, C. H. e Hodges, F. J. - Intracranial angiography. Radiology, 45:1-14, 1945.

16. Mount, L. A. - 'Treatment os spontaneous subarachnoid hemorrhage. J.A. M.A., 146:693-698 (junho) 1951.

17. Sugar, O., Holden, I. B. e Powell, C. B. - Vertebral angiography. Am. J. Roentgenol., 61:166-182 (fevereiro) 1949. 
18. Schwartz, H. G. - Arterial aneurysm of posterior fossa. J. Neurosurg., 5:312-316, 1948.

19. Poppen, J. L. - Diagnosis of intracranial aneurysms. Am. J. Surg., 75: 178-186, 1948.

20. Rosen, S. R. e Kaufmann, W. - Aneurysm of circle of Willis with symptom free interval of 27 years between initial and final rupture; report of a cese. Arch. Neurol. a. Psychiat., 50:350-354 (setembro) 1943.

21. Strully - Cit. por Mount 16 .

22. Murphy, J. P. - Surgical aspects of subarachnoid hemorrhage. M. Ann. District of Columbia, 18:119-128 (março) 1949.

23. Poppen, J. L. - Specific treatment of intracranial aneurysms; experiences with 143 surgically treated patients. J. Neurosurg., 8:75-102 (janeiro) 1951.

Galinger Municipal Hospital -- Washington 3, D.C., U.S.A. 